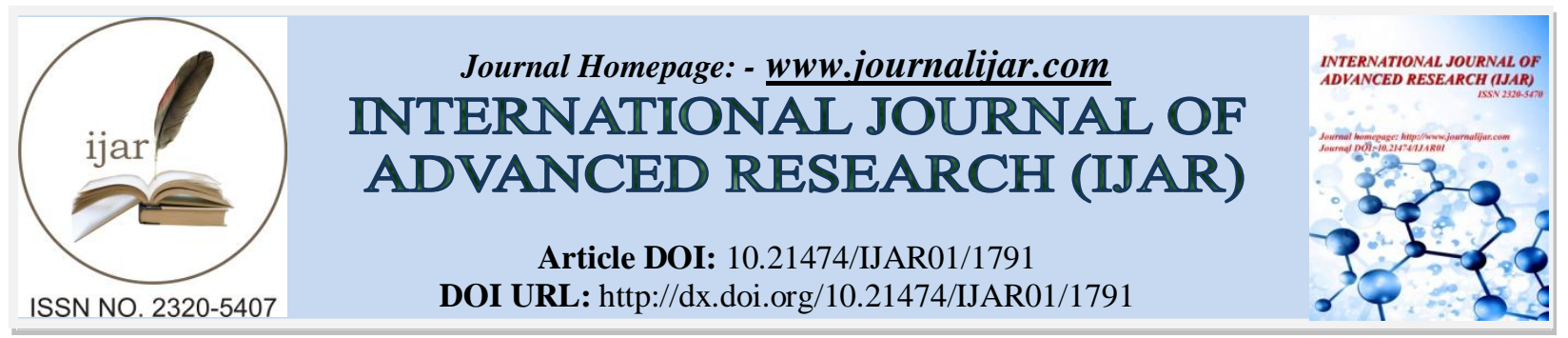

RESEARCH ARTICLE

\title{
RELATION BETWEEN (EPITHELIAL NEUTROPHIL-ACTIVATING PEPTIDE 78) OR (CXCL5) SERUM LEVEL AND CHRONIC LIVER DISEASES.
}

\author{
Jehan H Sabry ${ }^{1}$, Mohammed A El-Hendy ${ }^{1}$, Wafaa M El-sayed ${ }^{1}$, Amre M El Hammady ${ }^{2}$ and Noha W Abd \\ El-Rasheed ${ }^{3}$. \\ 1. Department of Clinical and Chemical pathology, Benha Faculty of Medicine, Egypt. \\ 2. Department of Internal Medicine, Benha Faculty of Medicine, Egypt. \\ 3. Department of Clinical pathology, Benha Psychiatric Hospital, Egypt.
}

\section{Manuscript Info}

(........................

Manuscript History

Received: 12 August 2016

Final Accepted: 22 September 2016

Published: October 2016

Key words:-

ENA78; chemokine; hepatocytes; Childpugh; Meld score.

\section{Abstract}

Background and aims:- CXCL5 is a small cytokine belonging to the CXC chemokine family predominantly expressed on epithelial cells . This cytokine stimulates the chemotaxis of neutrophils having angiogenic properties. Small interfering RNAs or antibodies against CXCL5 can suppress tumor growth.CXCL5 is considered a therapeutic target in liver diseases. The aim of our study was to assess CXCL5 serum levels in patients with chronic liver diseases.

Materials and Methods:- CXCL5 serum levels were measured in 60 patients with chronic liver disease and 20 apparently healthy controls. Results:- Serum CXCL5 levels in patients with liver cirrhosis were lower than in healthy controls, and correlated with the hepatic biosynthetic capacity, Child-Pugh and MELD scores. In patients with typical clinical complications of cirrhosis, CXCL5 levels were found to be decreased. CXCL5 levels were correlated with clinical presentation and laboratory parameters.

Conclusions:- serum CXCL5 levels are lower in patients with chronic liver disease, suggesting that CXCL5 might be involved in the pathogenesis of chronic liver disease. CXCL5 could serve as an additional biomarker for hepatic necroinflammation and fibrosis.

Copy Right, IJAR, 2016,. All rights reserved.

\section{Introduction:-}

Hepatic cirrhosis is an endstage disease and can be induced by several etiologies including viral hepatitis, biliary disease, autoimmune disorders and chronic alcohol abuse. ${ }^{(1)}$

Approximately 550 million people worldwide are chronically infected with hepatitis B (HBV) or hepatitis C (HCV) virus. The clinical route of $\mathrm{HBV}$ and $\mathrm{HCV}$ infection is variable ranging from chronic mild hepatitis to liver cirrhosis and hepatocellular carcinoma. ${ }^{(2)}$

Egypt commands the world in hepatitis $\mathrm{C}$ infection cases. Schistosomiasis and hepatitis $\mathrm{C}$ virus (HCV) co-infection is common in Egypt, Hepatitis B virus is also encountered. ${ }^{(3)}$ 
Although significant progress has been made to understand the molecular mechanism for advancement from acute liver injury to fibrosis and cirrhosis, there is no antifibrotic therapy actually available for patients with chronic liver disease. The mainstay is to treat the underlying liver disease. If this can not be performed and the patient progresses to decompensated liver cirrhosis, liver transplantation will be the only treatment option. ${ }^{(4)}$

Following an acute liver injury, hepatocytes are damaged and undergo necrotic or apoptotic cell death. This is joined with a massive inflammatory reaction, which is partly mediated by occupant kupffer cells or infiltrating inflammatory cells such as monocytes and neutrophils. ${ }^{(5)}$

Portal hypertension is the central cause of morbidity and mortality in liver cirrhosis. Complications of portal.$^{(8)}$ hypertension in cirrhotic patients include gastric and esophageal varices, ascites, portal hypertensive gastropathy, hepatorenal syndrome, hepatopulmonary syndrome and portopulmonary hypertension. ${ }^{(6)}$

Ascites is the most prevalent complication of cirrhosis and is connected with a poor quality of life, increased risks of other complications of cirrhosis such as; spontaneous bacterial peritonitis and hepatorenal syndrome with its high mortality rate the moment it is diagnosed. ${ }^{(7)}$

In those without underlying liver disease, $\mathrm{HCC}$ rarely occurs Chronic hepatitis B (CHB) represents approximately $50 \%$ of the implied etiologies for the expansion of hepatocellular carcinoma (HCC) worldwide. ${ }^{(9)}$

Hepatic stellate cells and other fibrogenic cell types are stimulated by chemokines and cytokines such as TGF-B1 to generate large amounts of extracellular matrix proteins. Several chemokines have been involved in the pathogenesis of liver fibrosis. For example the CCR2 ligand MCP-1 is produced by kupffer cells and HSCs, which enhances hepatic fibrosis by recruitment of macrophages that are associated with HSC activation. ${ }^{(10)}$

CXCL5 is well known for its function as chemoattractant and activator for neutrophils mainly during acute inflammatory responses , and it shares structural and biological features with IL- $8 .^{(11)}$

CXCL5 is produced by a diversity of cell types including monocytes, neutrophils, epithelial cells, fibroblasts and smooth-muscle cells, and its gene expression is generated by proinflammatory stimuli such as TNF, IL-1B and lps. ${ }^{(12)}$

Expression of CXCL5 is also increased in atherosclerosis but is not associated with neutrophil infiltration. Instead CXCL5 has a protective role in atherosclerosis by directly commanding macrophage foam cell formation. ${ }^{(13)}$

CXCL5 belongs to the CXC chemokine family and had been shown to have promitotic effects on hepatocytes. The aim of my study was to assess CXCL5 serum levels in patients with chronic liver disease.

\section{Patients and Methods:-}

Ethical consideration: Written informed consents were obtained from all participants, The study was designed as a case controlled prospective study after approval by the ethical committee of Benha Faculty of Medicine.

\section{Selection of subjects:-}

A total of 80 individuals were included in this study, who were categorized into the following groups:

Group 1:- sixty patients with chronic liver disease, who were selected from The Internal Medicine Department of Benha University Hospital between November 2014 and June 2015. Inclusion criteria were both males and females chronic liver disease patients aged more than 18 years while exclusion criteria were presence of Diabetes Mellitus, heart diseases or renal diseases.

Group 2 : Twenty apparently healthy subjects working as control group with normal ALT, negative HBV, HCV and HIV markers and with no history of liver disease. 


\section{Blood Sampling:-}

Peripheral blood was collected from each individual fulfilling antiseptic measures. The serum samples were separated by centrifugation at $3000 \mathrm{rpm}$ for $10 \mathrm{~min}$. then sera were stored at $-20^{\circ} \mathrm{C}$ till analysis. $1.8 \mathrm{ml}$ of blood for each $0.2 \mathrm{ml}$ trisodium citrate were used to separate plasma for PT and PTT measurement. K2EDTA blood samples were used for $\mathrm{CBC}$.

A) Standard parameters of liver and kidney functions as well as complete blood count and hepatitis markers were measured at Benha university hospital lab.

B) Estimation of scores:

Child stage:- The score employs five clinical measures of liver disease. Each measure is scored 1-3, with 3 indicating most severe derangement. ${ }^{(14)}$

\begin{tabular}{|l|l|l|l|}
\hline Measure & $\mathbf{1}$ point & $\mathbf{2}$ points & 3 points \\
\hline Total bilirubin, $\mu \mathrm{mol} / \mathrm{L}(\mathrm{mg} / \mathrm{dL})$ & $<34(<2)$ & $34-50(2-3)$ & $>50(>3)$ \\
\hline Serum albumin, $\mathrm{g} / \mathrm{dL}$ & $>3.5$ & $2.8-3.5$ & $<2.8$ \\
\hline Prothrombin time, prolongation $(\mathrm{s})$ & $<4.0$ & $4.0-6.0$ & $>6.0$ \\
\hline Ascites & None & Mild (or suppressed with medication) & $\begin{array}{l}\text { Moderate to Severe (or } \\
\text { refractory) }\end{array}$ \\
\hline Hepatic encephalopathy & None & Grade I-II & Grade III-IV \\
\hline
\end{tabular}

Chronic liver disease is classified into Child-Pugh class A to C, employing the added score from above.

\begin{tabular}{|l|l|l|l|}
\hline Class & A & B & C \\
\hline Points & $5-6$ & $7-9$ & $10-15$ \\
\hline
\end{tabular}

MELD score: MELD uses the patient's values for serum bilirubin, serum creatinine, and the international normalized ratio for prothrombin time (INR) to predict survival. It is calculated according to the following formula: $10 x(0.957 \log (\text { s.creat })+0.378 x \log (\text { T.Bil. })+1.12 \log (\text { INR })+0.643)^{(15)}$

\section{Measurement of serum CXCL5 concentrations by ELISA:-}

This was done using SunRed kit,provided by Biogene(e Mail:sunredbio@msn.cn).

\section{Test principle:*}

The kit uses adouble-antibody sandwich enzyme-linked immunosorbent assay (ELISA). To assay the level of Human Epithelial neutrophil activating peptide 78 in samples, (ENA-78/CXCL5) was added to monoclonal antibody enzyme well which was pre-coated with(ENA-78/CXCL5) monoclonal antibody, incubation; then, (ENA78/CXCL5) antibodies labeled with biotin, and combined with Streptavidin-HRP were added to form immune complex; then incubation and washing were carried out again to remove the uncombined enzyme. Chromogen Solution A\& B then added, the color of the liquid changed into the blue, and at the effect of acid, the color finally became yellow. The chroma of color and the concentration of the Human (ENA-78/CXCL5) of sample were positively correlated.

\section{Clinical complications:-}

Typical clinical complications due to portal hypertension, e.g. splenomegaly, ascitis, hypertensive gastropathy or esophageal varices, were evaluated by complete abdominal ultrasound and upper gastrointestinal endoscopy.

The medical history of gastrointestinal bleedings (variceal bleeding, hematemesis, melena, hemorrhoidal bleeding, intra abdominal bleeding and other serious bleedings) and the current systemic bleeding tendency unrelated to portal hypertension (epistaxis, prolonged post traumatic bleeding, gingival hemorrhage, spontaneous hematoma and hypermenorrhea / menorrhagia) were assessed by a standardized interview in all patients. 


\section{Statistical analysis:-}

The clinical data were recorded on a report form. These data were tabulated and analyzed using the computer program SPSS (Statistical package for social science) version 16. The significance of difference was tested using one of the following tests:- Student t test: Used to compare mean of variables in two groups of quantitative data. ANOVA test (F value): Used to compare mean of more than two groups of quantitative data. Inter - group comparison of categorical data was performed by using fisher exact test (FET). Correlation coefficient ( $r$ test): used to find relationship between two variables within the same group.

\section{Results:-}

CXCL5 serum concentrations are lower in patients with advanced liver disease

We evaluated 60 patients with chronic liver diseases. Serum CXCL5 levels in patients with chronic liver disease were significant lower than in healthy controls(p<0.043; Fig. 1). CXCL5 plasma levels decreased with the stage of cirrhosis according to the Child-Pugh classification (Fig. 2). The level of decompensation was also assessed by the MELD scoring system, and CXCL5 showed a negative correlation with the MELD score $(r=-0.418, p=0.001$; Fig.3). There was a significant correlation between CXCL5 serum levels and the hepatic biosynthetic capacity as assessed by various laboratory parameters such as albumin and prothrombin time (Table 1).

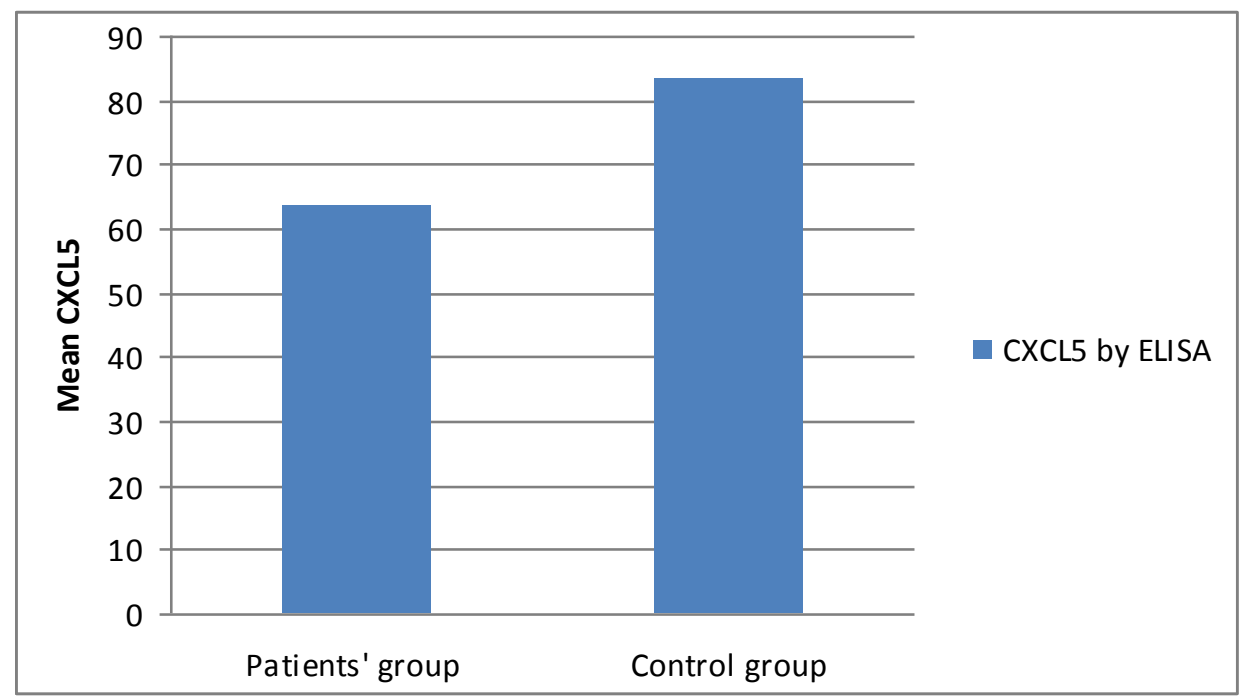

Fig. 1:- Comparison between patients' group and control group as regards serum CXCL5.

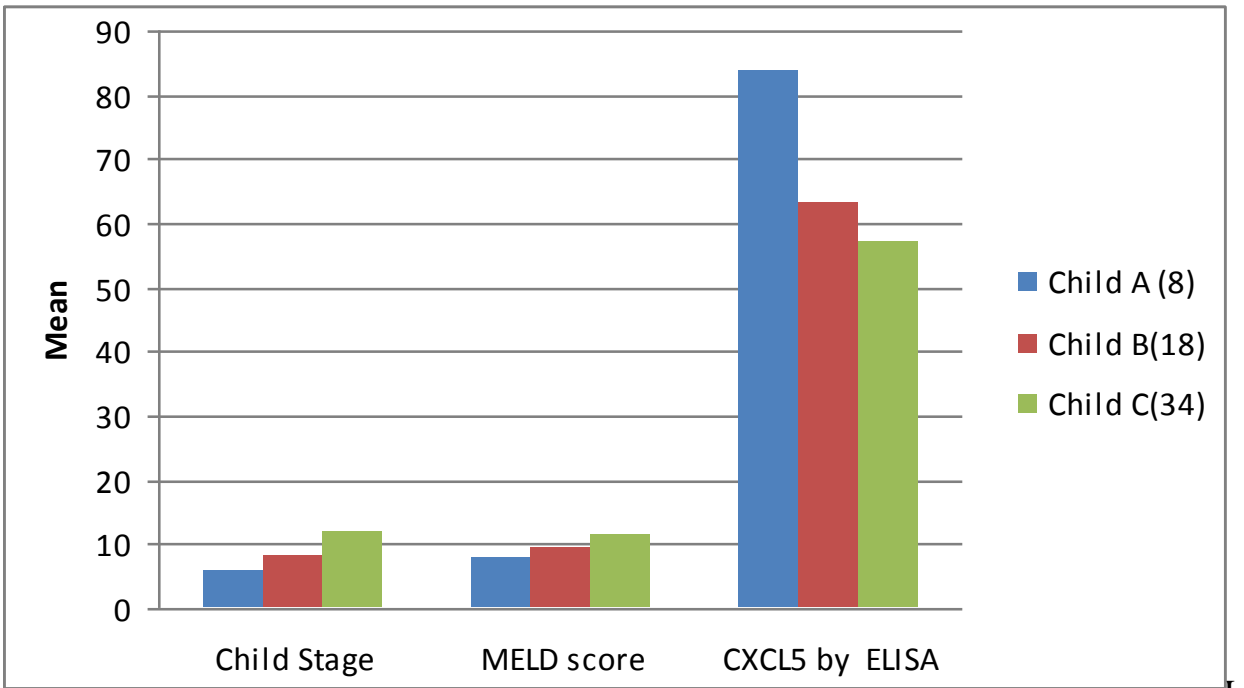

Fig. 2:- Comparrson detween panents suogroups as regards Cniru stage, iverd score and serum $\mathbf{A C L}$ 5. 


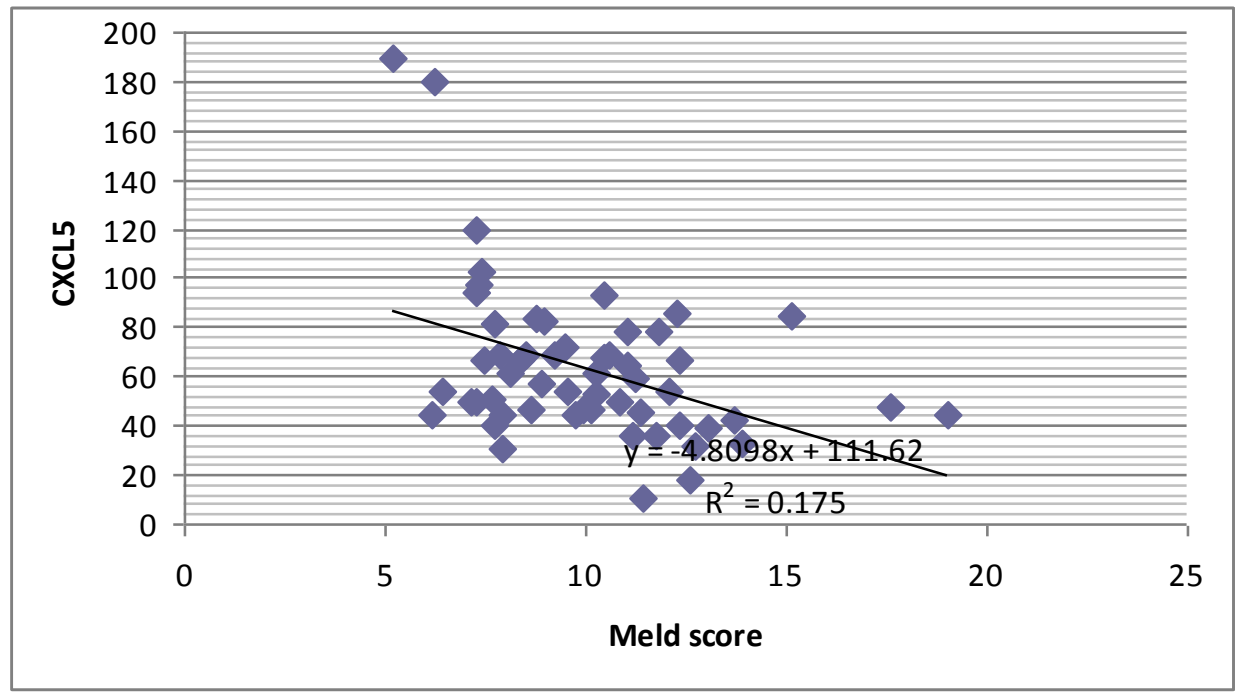

Fig.3:- Correlation between CXCL5 and MELD score among patients' group.

Table 1:- Correlation between CXCL5 and other parameters among patients' group.

\begin{tabular}{|l|l|l|}
\hline CXCL5 ELIZA & r test & P Value \\
\hline Age & 0.094 & 0.489 \\
\hline S.albumin $(\mathrm{g} / \mathrm{dl})$ & 0.443 & $0.001^{* *}$ \\
\hline S.creatinine $(\mathrm{mg} / \mathrm{dl})$ & 0.009 & 0.948 \\
\hline S.T.Bilirubin $(\mathrm{mg} / \mathrm{dl})$ & -0.384 & $0.003^{* *}$ \\
\hline Platelet count $(\mathrm{x} 1000 / \mathrm{mm})$ & 0.357 & $0.007^{* *}$ \\
\hline PT $(\mathrm{sec})$ & -0.382 & $0.004^{* *}$ \\
\hline aPTT $(\mathrm{sec})$ & -0.319 & $0.017^{*}$ \\
\hline INR & -0.386 & $0.003^{* *}$ \\
\hline Child Stage & -0.479 & $0.001^{* *}$ \\
\hline Meld score & -0.418 & $0.001^{* *}$ \\
\hline
\end{tabular}

\section{CXCL5 is lower in chronic liver disease patients with clinical signs of decompensation}

Patients with liver cirrhosis are susceptible to a variety of clinical complications such as splenomegaly, ascites, bleeding tendency, hypertensive gastropathy and esophageal varices. Patients with L.L. oedema \& HCC (Fig. 4A), Ascitis \& Jaundice (Fig. 4B), showed lower plasma levels of CXCL5. Thus, CXCL5 concentrations correlate well with clinical signs of decompensation.

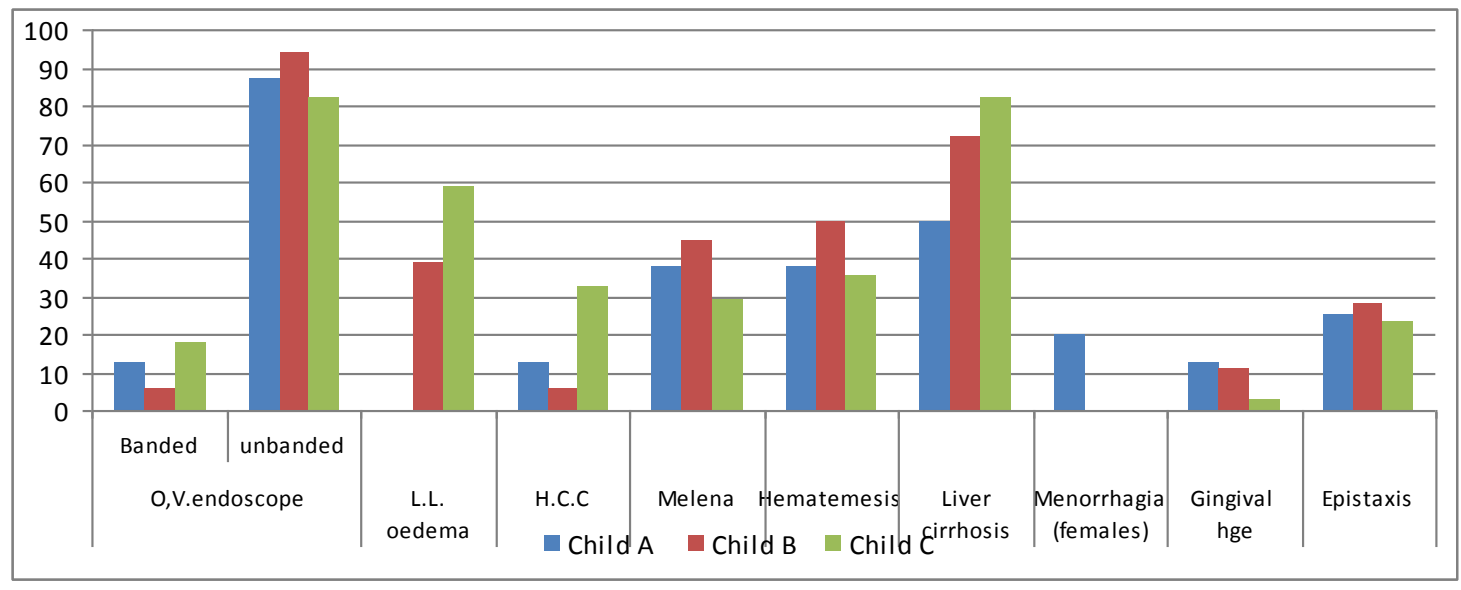

Fig.4A:- Comparison between patients' subgroups as regard clinical complications. 


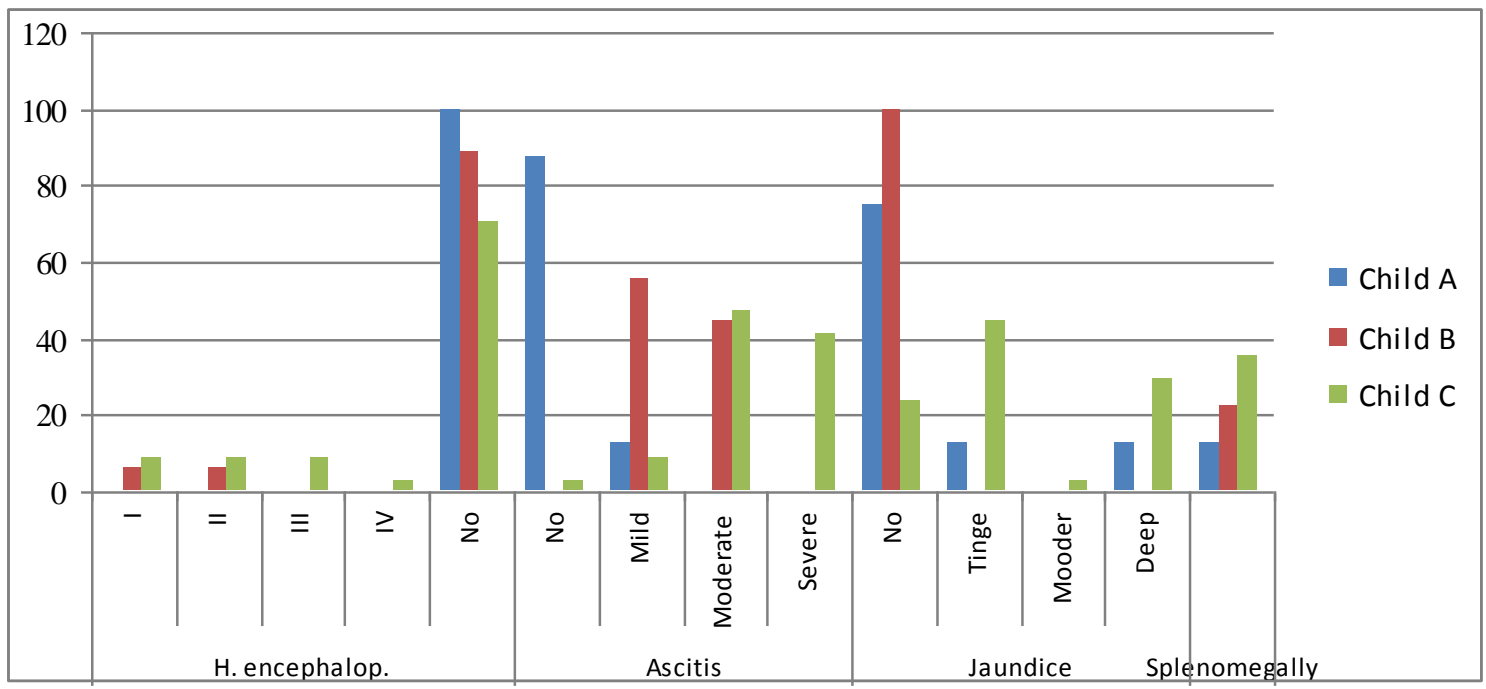

Fig.4B:- Comparison between patients' subgroups as regard clinical complications.

\section{Discussion:-}

Cirrhosis is a complication of liver disease which involves loss of liver cells and irreversible scarring of the liver. Hepatitis C, fatty liver, and alcohol abuse are the most common causes of cirrhosis of the liver in the U.S., Liver fibrosis is the scarring process that represents liver response to injury. Advanced liver fibrosis results in cirrhosis, liver failure, and portal hypertension and often requires liver transplantation. Cirrhosis is always developed from fibrosis. Although, fibrosis and cirrhosis are two different distinguished pathological conditions, they are closely related. ${ }^{(16)}$

The present study was designed to assess CXCL5 serum level in patients with chronic liver disease.

In this study, serum CXCL5 levels were reduced in patients with chronic liver disease compared with the control group.

These results were in agreement with Frank et al., ${ }^{(17)}$ who reported that plasma CXCL5 levels in patients with chronic liver disease were significantly lower than in healthy controls.

CXCL5 (also known as Epithelial neutrophil-activating peptide 78 or ENA-78) belongs to the CXC chemokine family and has been shown to have promitotic effects on hepatocytes. ${ }^{(18)}$

Patients with hepatic necroinflammation and fibrosis on liver histology showed lower serum level of CXCL5. In cirrhosis, CXCL5 levels were found to be decreased while Intrahepatically, CXCL5 expression was increased in patients with advanced fibrosis and cirrhosis. ${ }^{(17)}$

The isolation of different cellular compartments from mouse livers suggested ${ }^{\text {that }}$ hepatic stellate cells and sinusoidal endothelial cells are the main sources of hepatic CXCL5. ${ }^{(19)}$

Other cell types than hepatic stellate cells and endothelial cells must express and secrete higher amounts of CXCL5, as systemic plasma levels are lower in patients with chronic liver disease. Neutrophils and monocytes secrete CXCL5 and could be the extrahepatic source for systemic CXCL5.(20)

As the infiltration of blood monocytes and neutrophils into the liver participates in experimental hepatic fibrosis, the reduced circulating CXCL5 levels might be related to an altered distribution or activation of these innate immune cell subsets in patients with advanced liver disease. ${ }^{(5)}$

Plasma CXCL5 levels are lower in patients with chronic liver disease, suggesting that CXCL5 might be involved in the pathogenesis of chronic liver disease. CXCL5 could serve as an additional biomarker for hepatic necroinflammation and fibrosis. ${ }^{(17)}$ 
In the present study, reduction in CXCL5 serum levels in chronic liver disease patients was associated with alteration in the hepatic synthetic capacity.

There was a high statistically significant decrease of serum albumin in patients' group when compaired with control group and in different stages of cirrhosis than in control group while there was statistically highly significant increase of (PT and aPTT) in patients' group than in control group and in different stages of cirrhosis than in control group.

Correlation studies revealed that, CXCL5 serum concentration seems to correlate with other parameters among patients' group. There was significant positive correlation between CXCL5 and serum albumin and there was significant negative correlation with (PT and aPTT) among patients' group.

These results go in hand with Essam et al., ${ }^{(21)}$ who reported that serum albumin level in patients with chronic liver disease were significant lower than in healthy controls. And with Mohammed et al., ${ }^{(22)}$ who reported that PT, aPTT, INR were significally increased in all patients with liver diseases and platelet count showed significant decrease among cases.

Decrease levels of serum albumin in chronic liver diseases was explained by decreased synthesis by the hepatocytes in addition to water and sodium retention that dilutes the content of albumin in the extracellular space. Other factors likely contribute to the development of hypoalbuminemia, includes an increased transcapillary transport rate. ${ }^{(23)}$

Patients with decompensated liver cirrhosis have significantly impaired synthetic function. Many proteins involved in the coagulation process are synthesized in the liver. ${ }^{(24)}$

Liver production of coagulation factors 1, 2, 5, 7, 9 and 10 which is reduced in chronic liver disease. Factors 2, 7, 9 and 10 are further reduced by vitamin $\mathrm{K}$ deficiency due to cholestasis.PT can be selectively elevated because factor 7 is the first factor to be depleted in cirrhosis due to its short half-life..$^{(25 \& 26)}$

Result of the present study showed a high statistically significant decrease of platelet count in patients groups when compaired with the control group with gradual decrease in different stages of cirrhosis and positive correlation with CXCL5.

liver production of plasma CXCL5 is mainly platelet-derived, suggesting the role of platelets in immune responses and inflammation. Measurement of CXCL5 in human blood allowed testable inferences concerning physiology and pathophysiology in quantitative platelet disorders. CXCL5 was reported to be secreted by peripheral blood monocytes and involved in neutrophil activation. Interestingly, CXCL5 also share similar functions in platelet activation and coagulation. As platelet alpha-granules contain a variety of growth factors, cytokines, and chemokines, in addition to hemostatic proteins, platelets might be a source of CXCL5. ${ }^{(27)}$

Result of present study showed a highly significant increase of serum total bilirubin in patients' group than in control group with gradual increase in different stages of cirrhosis showing negative correlation with CXCL5.

In this study Patients without liver cirrhosis had significantly lower levels of CXCL5 than controls, but higher levels than patients with Child A-C cirrhosis . CXCL5 serum concentrations correlates with Child- Pugh's stage of liver cirrhosis. And also with the disease severity as assessed by the MELD score.

As regard signs of decompensated liver cirrhosis there was statistically significant increase in the percentages of L.L oedema, HCC, ascitis and jaundice in patients' group as regards different stages of cirrhosis and patients with liver cirrhosis who had manifestation of hepatic decompensation showed lower plasma levels of CXCL5.

In consistent with these findings Frank et al., ${ }^{(17)}$ revealed that CXCL5 concentrations correlate well with clinical signs of hepatic decompensation.

Edema in patients with cirrhosis has a multifactorial pathogenesis. The most obvious pathogenic mechanism is portal hypertension causing increased hydrostatic pressure within the mesenteric veins and causing fluid to seep out into the mesentery. In addition, liver dysfunction produces a state of water overload due to hypoalbuminemia and 
decreased aldosterone catabolism. This also plays a role in producing mesenteric edema, ascites, pleural effusions, and subcutaneous edema. ${ }^{(28)}$

Statistically significant relation between the mean serum CXCL5 level and HCV as cause of cirrhosis was reported in the results of the present work.

Studies conducted at National Liver Institute (NLI), $\left.{ }^{(29} \& 30\right)$ reported that hepatitis C was the most frequent cause of chronic liver disease in Egypt. Hepatitis $\mathrm{C}$ antibodies were more common in patients with active schistosomiasis and patients without hepatitis B surface antigenaemia. These studies found that $60 \%$ of patients of chronic liver disease were anti-HCV positive and $40 \%$ had $\mathrm{HCV}$ viremia, as compared with only $6 \%$ having $\mathrm{HBsAg}$ and $8 \%$ having S. mansoni ova, so the predominant cause of chronic liver disease in NLI outpatients was infection with HCV.

In conclusion, plasma CXCL5 levels are lower in patients with chronic liver disease. Decreased CXCL5 concentrations appears to be a good marker for cirrhosis and its staging, clinical signs of decompensation and synthetic capacity of the liver. further studies are required to determine if CXCL5 supplementation could be used as therapeutic intervention in chronic liver disease and fibrosis.

\section{References:-}

1. Reham J, Taylor B, Mohapatra S, Baliunas D, Patra J and Roerecke M. (2014): Alcohol as a risk factor for liver cirrhosis: a systemic review and meta - analysis. Drug Alcohol Rev. ; 29: 437- 445.

2. Panther $\boldsymbol{E}$ and Thimme R. (2012): "Therapy of chronic hepatitis B and C virus infections in the clinical practice". Praxis ; 101(1): 37- 42.

3. El-Sabah A.A, El-Metwally M.T and Abozinadah N.Y. (2011): " Hepatitis C and B virus in schistosomiasis patients on oral or parenteral treatment". J Egypt Soc Parasitol.; 41(2): 307- 314.

4. Abdelwahab SF, Hashem M, Galal I, Sobhy M, Abdel-Ghaffar TS, Galal G, Mikhail N, El-Kamary SS, Waked I and Strickl and GT (2013): "Incidence of hepatitis C virus infection among Egyptian healthcare workers at high risk of infection". J Clin Virol. Pii: S1386-6532.

5. Karlmark KR, Weiskirchen R, Zimmermann HW. (2014): Hepatic recruitment of the inflammatory Grl + monocyte subset upon liver injury promotes hepatic fibrosis. Hepatology; 50: 261 - 274.

6. Ashkenazi E, Kovalev $Y$ and Zuckerman E. (2013): "Evaluation and treatment of esophageal varices in the cirrhotic patient". sr Med Assoc J.; 15(2): 109-115.

7. Radeleff B, Stampfl U, Sommer C., Bellemann N., Hoffmann K., Ganten T., Ehehalt R. and Kauczor H. (2012): "Transarterial ablation of hepatocellular carcinoma : Status and developments". Radiologe; 52(1): 4455.

8. Ha N, Ahmed A, Ayoub W., Daugherty T., Chang E., Lutchman G., Garcia G., Cooper A., Keeffe E. and Nguyen M. (2012): "Risk factors for hepatocellular carcinoma in patients with chronic liver disease: a casecontrol study". Cancer Causes Control; 19.

9. Kim B, Han K and Ahn S. (2011): "Prevention of hepatocellular carcinoma in patients with chronic hepatitis B virus infection". Oncology ; 81 Suppl 1:41- 49.

10. Tatianna K, David B, et al., (2014): Interleukin -17 Signaling Inflammatory cells Kupffer, and Hepatic Stellate cells Exacerbates liver fibrosis in mice. Hepatology and Gastroenterology.

11. Frank T, Menning W, Zimmermann, et al., (2013): Structure and neutrophil-activating properties of a novel inflammatory peptide (ENA-78) with homology to interleukin 8.J Exp Med.; 174: 1355- 1362.

12. Kubes P, Mehal WZ, (2013): Sterile inflammation in the liver.Gastroenterology; 143: $1158-1172$.

13. Rousselle A, Qadri F, Leukel L, Yilmaz R, Fontaine JF, Sihn G, et al., (2013): CXCL5 limits macrophage foam cell formation in atherosclerosis. J Clin Invest 123:1343-1347.

14. Cholongitas E, Papatheodoridis GV, Vangeli M, Terreni N, Patch D, Burroughs AK. (2005): Systematic review: The model for end-stage liver disease-should it replace Child-Pugh's classification for assessing prognosis in cirrhosis? Alimentary Pharmacology\& Therapeutics 22(11-12): 1079-1089.

15. Kamath PS, Wiesner RH, Malinchoc M, Kremers W, Therneau TM, Kosberg CL, et al., (2001): A model to predict survival in patients with end-stage liver disease. Hepatology, 33: 464-470.

16. DALLAS, Lefton HB, Rosa A, Cohen M. (2015): Diagnosis and epidemiology of cirrhosis. Med Clin North Am.; 93(4): 787-799.

17. Frank Tacke, Henning W. Zimmermann, Christian Trautwein, and Bernd Schnabl. (2011) : CXCL5 plasma levels are decreased in patients with chronic liver disease. J Gastroenterol Hepatol.; 26(3): 523-529. 
18. Dominguez M, Miquel R, Colmenero J, Moreno M, Garcia-Pagan JC, Bosch J, Arroyo V, Gines P, Caballeria J, Bataller R. (2009): Hepatic expression of CXC chemokines predicts portal hypertension and survival in patients with alcoholic hepatitis. Gastroenterology; 136(5):1639-1650.

19. Taura K, De Minicis S, Seki E, et al., (2008): Hepatic stellate cells secrete angiopoietin 1 that induces angiogenesis in liver fibrosis. Gastroenterology; 135: 1729-1738.

20. Walz A, Schmutz P, Mueller C, Schnyder-Candrian S (1997): Regulation and function of the CXC chemokine ENA-78 in monocytes and its role in disease. J Leukoc Biol.; 62: 604-11.

21. Essam F. Al-Jumaily and Faiha'a M. Khaleel (2012): The Effect of Chronic Liver Diseases on Some Biochemical Parameters in Patients Serum. Current Research Journal of Biological Sciences; 4(5): 638- 642.

22. Mohammed Elamin Mustafa1, Mansoor Mohammed Mansoor and Asaad Mohammed Ahmed Abd Allah Babker (2015): Evaluation of platelets count and coagulation parameters among patients with liver disease. World Journal of Pharmaceutical Research SJIF Impact Factor; 7105 - 2277ISSN Research Article.

23. Mauro Bernardi, Henricksen JH, Siemssen O, Krintel JJ, Malchow-Moller A, Bendtsen F, Ring-Larsen H. (2001): Dynamics of albumin in plasma and acitic fluid in patients with cirrhosis. J Hepatol ; 34:53- 60 .

24. Pooja D. Amarapurkar, S. Sherlock and J. Dooly. (2011): "Haematology of liver disease," in Diseases of Liver and Biliary System, S. Sherlock and J. Dooly, Eds., pp.; 43-62, Blackwell Science, London, UK, 10th edition.

25. Jacob A.Udell , O'Brien DP, Shearer MJ, Waldron RP, Horgan PG. (2012): The extent of vitamin K deficiency in patients with cholestatic jaundice. $J$ R Soc Med.; 87(6): 320- 322.

26. Jacob A.Udell , van Dam-Mieras MC, Hemker HC. (2012): Half-life time and control frequency of vitamin K-dependent Coagulation factors. Haemostasis; 13(3): 201- 208.

27. Xingmin Feng, Gear AR, Camerini D. (2012): Platelet chemokines and chemokine receptors: linking hemostasis, inflammation, and host defense. Microcirculation; 10: 335- 350.

28. Shailendra Chopra, Gines P, Fernandez-Esparrach G, Arroyo V, Rodes J. (1999): Pathogenesis of ascites in cirrhosis. Semin Liver Dis.; 17:175-189.

29. Waked I, Saleh S, Moustafa M et al., (1995) : "High prevalence of hepatitis C in Egyptian patients with chronic liver diseas". Gut; 37: (1)105-107.

30. Strickland G, Elhefni H. and Salman T. (2002): "Role of hepatitis C infection in chronic liver disease in Egypt". Am J Trop Med Hyg.; 67(4): 436-442. 